\title{
Networking: a study in planning and developing cross-cultural collaboration
}

\author{
Sanjeev Singh* and Jon Dron** \\ *Institute of Informatics and Communications, University of Delhi South Campus \\ **School of Information Management, University of Brighton \\ email:Sanjeev@iic.ac.in, Jon.Dron@bton.ac.uk
}

This paper reports on a collaboration between the authors at the University of Brighton (UK) and the University of Delhi, South Campus. The collaboration came about as a result of the EU-India Cross-Cultural Innovation Network collaboration programme, a project involving several universities and organizations across Europe and India. The authors of this paper both lecture in the area of computer networking. Following meetings in Delhi, they agreed to work together to produce a Web-based networking resource to be generated by the students of both institutions. The first phase of development involved the mounting of Web-based tutorials and documents produced by the students. The second phase will centre on the development of a knowledge base generated by the interaction of the students within an asynchronous forum. Running alongside these phases will be a collaborative bookmarking system, a database in which the students will post URLs of Web-based resources that they find useful in their studies. This system incorporates a form of collaborative filtering, an evolutionary mechanism which seeks to embody the qualities that students value in resources to provide a dynamic set of ratings to assist in the selection of those of most use. The planning of such a system raises some unusual issues, not least in the process of collaboration itself, with concerns as diverse as technical compatibility, institutional and cultural differences, timezones and the reliability of email. Limited bandwidth between our institutions causes special problems with the interactive elements of the resource. We present the methods we are investigating to reduce the impact of this. The fact that the students share an intellectual discipline but are otherwise separated by a cultural and geographical divide is expected to lead to fruitful diversity in thinking and approaches to problem-solving.

\section{Introduction}

This paper discusses the theoretical and practical issues of implementing a Web-based learning collaborative project across cultural borders. The overall purpose is to convey to 
the student community the benefits of incorporating a Web-based cross-cultural learning environment into a conventional course. The criteria for successful applications are presented and issues relating to training and design, are discussed.

The value of extending the classroom beyond national boundaries is only one of the many reasons for increased intercultural communication relating to the growing internationalization of education (Brislin and Yoshida, 1994). On graduation students will often be required to interact with others from different cultural backgrounds, whether they are well prepared to do so or not. In the hope of building strong international working relationships between people and organizations of different cultures, it is important that students be prepared for the emerging future where cross-cultural communication skills will be of great importance. One way of developing these abilities is to present students with authentic opportunities to work with people who possess specialized skills in a shared subject domain.

The sociocultural context in the present model includes different values, learning environments and cultural/social perspectives of learners in two or more countries; these concerns are at the root of many problems that arise in international or cross-cultural programmes (Conner, 1990). An analysis of cultural issues shows that the predominant trend in the area is to ignore the unique cultural contexts of diverse learners. Occasionally, instructional adaptations and modifications are made to enhance cultural appropriateness, but cultural subtleties and deep-rooted value systems are often neglected in this process. It is nonetheless essential to take into account both the deep structure (values, learning styles) and the surface level (spoken language, forms of interaction) dimensions of a culture.

Each country manages its own educational system and establishes its own priorities for training and design. A 'not-invented-here' mentality has made many established institutions unwilling to use or recognize courses created by other institutions or in other countries. Funding policies and mission clarity and articulation also become more complex when carried across national borders (Murphy, 1991). Thus the development of a crosscultural course must be sensitive to these issues in order to succeed.

\section{Rationale}

Web-based training does not merely provide a different medium for traditional classroom interactions, but creates an environment for students to take part in much richer and relevant educational activities. By incorporating a Web-mediated cross-cultural learning activity into the course curriculum, students may be presented with new and greater challenges that extend beyond their traditional, and often passive, learning of theories and content. With respect to building cultural awareness and tolerance, Web-mediated crosscultural learning activities have the potential of bringing about behavioural and attitudinal changes in people that otherwise would not occur. For example, students may develop a deeper sensitivity for culturally based differences in work habits by interacting with students of diverse cultures rather than reading about how one should be culturally aware of these differences in textbooks. Likewise, pre-service teachers may acquire a better understanding of culturally based differences in learning styles, knowledge representation and cognition if they are given the opportunity to interact with students from diverse cultures. 
Perhaps more important, however, is that providing students with the opportunity to participate in an authentic cross-cultural exchange releases them from the proverbial academic vacuum in which they typically build knowledge and understanding from readings and in-class discussions (Dron, Mitchell, Siviter and Boyne, 2000). Computer communication technology has already changed the image of the classroom. These networks stretch across international borders to form cross-cultural learning environments where teachers and students of diverse cultures can learn with and from each other. With its capability of bringing innovative views to the classroom instantaneously, this collaborative project presents students with new opportunities to develop cultural awareness and build shared knowledge.

Some of the important criteria for implementing our Web-based cross-cultural learning project are that the collaborative project must:

- be relevant to the course and integrated into the curriculum goals;

- include some aspect of work or learning that could not be accomplished without crosscultural computer mediation. Student interest in, and commitment to, the virtual learning environment may diminish if they perceive that the learning activities could be done just as easily, if not better, outside the international forum. It is crucial that the cross-cultural model contains a unique element or dimension that otherwise would not be present;

- have a clear and definite time-frame. Students aware of the time-frame within which they are working may be more inclined to adopt proactive writing habits so that the virtual learning environment is not adversely affected by prolonged inactivity;

- be as simple and uncluttered as possible. Overly complex activities may lead to information overload, resulting in frustration, loss of interest and inactivity;

- be preceded with greetings and introductions. Students should be given the opportunity to 'meet' online before engaging in the structured learning activity;

- encourage information-sharing and knowledge-building. One of the greatest potentials of a Web-mediated cross-cultural exchange is to overcome the barriers that restrict the flow of ideas and the sharing of knowledge;

- avoid compromising learning quality. Although it may provide new and innovative learning opportunities, the academic standards and levels of achievement must be maintained.

Other advantages of this collaborative project are that, like many electronic methods of learning, it introduces new opportunities for collaborative learning, facilitates individualized feedback as well as contact with peers and faculty, promotes reflective and critical thinking due to its asynchronous nature, and permits students to work at a time and pace that is convenient to them. Web mediation can bring these benefits to a learning environment; cross-cultural applications of this approach therefore have the potential to be extremely useful and relevant to students' learning. Therefore, it is more than worthwhile to review current curriculum goals for viable opportunities for Web-mediated crosscultural learning activities. 


\section{Context}

\section{The Indian context}

Pedagogical value in India is directed towards acquiring knowledge in the form of information disseminated by the elders in general, and the teacher in particular (Jadhav, 1999). Mutual (student-to-student) interaction is less usual, typically being confined to exchange of notes and issues relating to interaction with the management of the teaching programme. Students in India place a high value on the amount of effort one puts in acquiring knowledge through textbooks. Emphasis on hard work is also reflected in heavy daily teaching schedules fixed by the system. Students are always busy in finishing their assignments rather than looking for other resources. There is little relaxation time or flexibility in which to consult other resources because of the time constraints.

With the increasing academic competition and pressure on the students to perform well, the education system today encourages earning grades. This is what students concentrate on: whatever is taught is simply absorbed by them, memorized and then reproduced at the time of examination.

The university campuses generally do not provide adequate hostels or dormitory facilities. Students are geographically dispersed. This prevents an educational institution from simply establishing its own Internet and Web service department and forces the educational institution to rely extensively upon ISP. Thus, it is very difficult to implement such networks in the majority of universities and higher education institutions. These are essentially maintained by individuals.

The shortage of Internet bandwidth, spurred by increased Net usage, poses a major constraint in India. The current domestic traffic demands a minimum throughput of 4 Gbps, which at present is serviced by an international pipe with a dismal capacity of around $420 \mathrm{Mbps}$. Already deficient by 88 per cent, the bandwidth used by institutions in India in general is of the order of $64 \mathrm{kbps}$ to $128 \mathrm{kbps}$. This is one of the major technical problems in popularizing Web-based learning models in India. Power backup is also a problem, so the typical server downtime is greater than in the UK. The online discussion groups are also limited by the time constraints. Although students may want to join these discussion groups, the institute may be closed and facilities do not exist at home.

\section{The UK context}

The course in Delhi receives around 2,000-2,500 qualified applicants each year for 35 places. It is thus unsurprising that the quality of the students is exceptionally high when compared with the more modest ratios of the University of Brighton. There are also significant differences in class size, with a modular system in Brighton teaching up to 150 students in lectures and tutorials at undergraduate level as opposed to the small, focused cohort of 35 students in India. Although the motivation of UK students is often very high (particularly in postgraduate courses) there is a higher proportion of less able and less willing students, as well as greater difficulties providing personal care and attention from faculty staff. Partly as a result of this, as well as through the active efforts of teaching staff and a slightly less rigidly hierarchical social ethos, there is a greater tradition of spontaneous group working and mutual assistance amongst students in this institution.

Although technical provision is generally significantly better in the UK than in India, 
students must share resources with a far greater diversity and number of colleagues than their Indian counterparts, who effectively have a dedicated PC for their own use. Internet usage is also somewhat restricted due to constraints placed by JANET and the conflicting needs of different groups of users within the university. For example, use of Internet Relay Chat (IRC) is explicitly banned. However, the vast majority of students of computing not only have a PC of their own but also a connection to the Internet with a private ISP, so it is far simpler for them to make use of the Net- and Web-based resources to allow flexible working. This extra time helps to compensate for the somewhat lower innate abilities of the majority of students.

The ethos in Brighton is only slightly more relaxed than that in Delhi with regard to timetabling and teaching methods, and similar constraints exist to those in India. There are, for example, few ways that provision is made for the creation and use of the online teaching in the allocation of staff resources. Both institutions are still culturally based in a traditional model of lectures, tutorials and workshops. Although this is changing as a result of pressures from within and without the universities, it provides a greater set of obstacles than would be the case if online learning were a more integrated part of the overall system.

\section{Methodology}

A prime concern is that students be adequately trained to use the system so that any anxieties or apprehensions about using the technology are overcome by the time the crosscultural Web-mediated learning activity begins. This entails building sufficient practice time into the course curriculum.

A prototype Website has been launched on one of the servers of the University of Brighton (EU-India prototype Website, 2000). This resource is built by and for students of the universities of Delhi and Brighton. Its purpose is to provide a shared resource on the subject of networking for students in India and the UK. A Brighton-Delhi discussion group has also been created and students are strongly encouraged to interact with their collaborative institute and to comment on the resources. It was important to identify common ground without diminishing the importance of cultural differences. Both cultural-general and cultural-specific approaches to designing the virtual space and learning activity were considered.

Various articles and tutorials were chosen to cover the common course curriculum of both the universities. These were uploaded and classified to help students structure their learning. Online tests were also mounted for self-assessment. The students were asked to share their knowledge and queries using the mechanism presented on the site.

Students were also asked to prepare a database of resources (URLs, notes and suitable links) based on their mutual interaction. This was enabled using a Web application (CoFIND) developed by one of the authors (Dron, Mitchell, Siviter and Boyne, 1999). CoFIND is designed to discover what sorts of metadata are valued by its users. Learners enter qualities, the things about a resource which are considered valuable, such as 'good for beginners', 'detailed', 'clear' and so on. These qualities are then used to rate resources that the learners also add to the system. As such, it presents a useful opportunity to identify the aspects of learning resources valued by groups of learners. 


\section{Findings}

\section{Pragmatic issues}

Selecting groups of students with similar interests was not too difficult, as the basic curricula across the institutions are quite similar. However, this was far from being the end of the problem. Students in India generally follow a direct path from undergraduate to postgraduate qualifications as a matter of course, whereas this is relatively rare in the UK. It was decided that the closest match of curricula and size/type of course was between the postgraduate part of the course in India and a conversion masters course (M.Sc. in Information Systems) at the University of Brighton, notably in the area of networking technologies. Although the curricula for these courses are similar, they are at slightly different ends of the soft-hard divide, with Indian students more concerned with the engineering side and British students more involved in the world of organizations and systems. This difference in emphasis was felt to be potentially valuable, allowing the strengths of each group of students to counterbalance the weaknesses of the other.

Both courses cater for a group of $30-40$ postgraduate students. However, as the UK masters' course is a conversion degree, students are usually at a significantly lower technical level than their Indian counterparts at the start of the course. To make matters worse, semesters in India start in July as opposed to October in the UK, exacerbating the gulf between knowledge levels. After much investigation of options, we were eventually able to identify a subject and time slot suitable for both groups of students near the start of the second semesters in each institution, which would allow us to apply our requirement for a clear time-frame. This left us with the problem of how to introduce students to each other and engage them in mutually beneficial interaction prior to sharing experience and understanding. The first route to achieving this was through the use of the collaborative bookmarking system. Using this, students are able to contribute in an anonymous and beneficial way, with the ability to find resources only loosely related to computing ability. It is a system where clear benefits can be achieved through the efforts of a larger, more diverse group.

We felt that it was important to start the socialization process earlier than any significant collaboration effort. As such, we created a simple discussion mechanism and relied on students' curiosity to start it moving. Unfortunately, the difference in semester start dates resulted in disappointment for early adopters. A re-launch was planned to make sure students were informed of the resource in each location. However, even then little use was made of this. Students in both institutions tend to organize their time according to the need to achieve good assessment results rather than out of pure curiosity. As there was little pressure applied by teachers, and as it was not clear to the students what the benefits would be, it was not heavily used. We were also unable to establish the clear and definite time-frame that we had originally identified as a requirement.

\section{Technical issues}

The initial site that was set up to provide a basis for discussion was built using off-the-shelf components (such as a Web-based discussion forum) available as part of Microsoft's FrontPage. Although the tool contains some assistance for group working, bandwidth constraints meant that it was not feasible to work together effectively on a single live site. However, it provided a simple means to get a static set of pages up quickly. Both 
institutions employ a wide variety of technologies for Web serving, from Apache under Solaris to Microsoft's IIS under NT to Lotus Domino. Of these, Domino seemed the logical choice for the discussion forum due to its ability easily to replicate databases, although bandwidth issues continue to make this a lengthy process. However, costs for extra client licences were high and our budgets were low so the current iteration of the environment makes use of TalkItOver (http://www.talkitover.com), an environment which provides the benefits of both bulletin boards and mailing lists in a single (albeit slightly slow) package, equally accessible to Indian and British students. We are still considering opting for the tried and tested technology of Usenet News which, despite its simplicity, provides most of the features needed in a discussion environment and is more usable due to its familiarity and built-in support for replication. However, technical limitations on propagation of newsgroups at the University of Brighton have made this hard to implement.

We had considered the possibility of synchronous discussions, but the time-zone difference meant that it would be difficult to find times that would fit the timetables of each group of students and we were keen to make it as simple as possible to communicate.

CoFIND, the collaborative bookmarking system, was already written using Microsoft's ASP. Initially two separate systems were installed at each location, but an unfortunate series of technical problems in Delhi (where uninterruptible power supplies need to be the size of large freezers and temperature control is a serious issue) led to the loss of all our accumulated data. The bandwidth requirements of the application were not particularly high so it was felt to be unnecessarily difficult to replicate this across both servers. The system has now been implemented on a single server in the UK. However, it has mainly been used by Brighton students, which is felt to be the result of the enthusiastic selling of the system by its author.

\section{Working together}

After quite brief initial meetings in Delhi, most of the time spent on discussing the process has involved exchanges of email between the authors of this paper. We met in the UK for further discussions later the same year, and again in Delhi the following year. It is highly significant that most advances have been made as a result of our face-to-face meetings. A lot of the reason for this is to do with the fact that such meetings provide a focused period when all we need to do is to work on the system. However, it is interesting to observe the amount of time spent on getting to understand each other's motivation and expectations, as well as clarifying meanings.

\section{Discussion}

\section{The experience in India}

In spite of the common view of pedagogy as knowledge acquisition, the main problem encountered during the course was technical. It was observed that students were willing to use the mechanism set up in the project but they were restricted by the system. Although students may want to join the discussion groups that were set up, for example, the institute was often closed and facilities do not exist at home. This certainly had some effect on lack of momentum sustained in discussions, requiring significant commitment to stay involved. Given the students' other priorities, this commitment was generally not achieved. 


\section{The experience in the UK}

It was noted at the outset that Brighton's support for materials development was oriented to traditional forms of teaching and learning. This posed problems for the collaboration. As Moore and Kearsley note, development of distance learning in a piecemeal fashion is unsatisfactory from a student and a developer perspective (Moore and Kearsley, 1996). These problems are exacerbated when trying to integrate materials and resources from two culturally and geographically isolated institutions, with no shared systems.

As with the Indian students, another major problem was in making students' contributions to the joint effort worthwhile in terms of process and reward. Our solution was twofold. Firstly, we used the time-honoured trick of making participation a requirement to achieve marks. This is not as satisfying as it might be and much work is still needed to make it desirable to make use of the system as a means to achieving other ends, rather than as an end in itself. We therefore put a lot of effort into making the resources as useful as possible. Tutorials unavailable elsewhere (many created by previous years' students as projects) were posted and a lot of emphasis was placed on the importance of finding Web-based help and posting it to the collaborative bookmark system. This failed to reach the critical mass needed to make it self-sustaining but was used by some students to help them with individual projects and dissertations.

\section{Conclusions}

We believe that whilst we have developed a workable model for collaboration, we are not yet in a position to report significant success in this venture. Although we have been planning and running parts of the system for nearly two years, progress has been slow and fitful. Part of the reason for this lies in attempting to add the collaboration to our already full timetables, but we have also encountered a host of problems from the technical (for example, Delhi's mail server was unavailable for several weeks and an experimental bookmark database was lost to a rogue machine) to the practical (for example, the fact that students in Delhi started just as students in Brighton finished) to the cultural (coming to terms with different approaches and another ethos).

It is significant that most progress has been made when the authors have been able to meet in person. Partly this is due to the need to focus on the issues at hand in a limited timeframe, but it also highlights the need for constant checking of cultural cues when discussing a wide range of issues, and the inadequacy of electronic means of communication to achieve this.

For reasons already cited, this attempt to collaborate has not been as successful as we had wished. Technical issues will soon be less important as the reliability and speed of networked connections improves. However, maintaining the momentum of the project has proved to be an intractable problem. Our latest solution is to provide students with assignments and projects requiring the development of tutorials which will be of use to other students in each country. We then collate this growing resource base, resulting in a form of collaboration without the students needing to try too hard. Although this is far from our original intentions of enabling students to work together, it provides a low threshold process whereby the strengths of students in each institution may be shared and may provide the glue to enable more successful collaboration in future. This part of the 
collaborative project has motivated students to build their own Web-based educational resources and now these are maintained by the students in India, including the content development, updating and so on (http://services.iic.ac.in).

\section{Acknowledgements}

Authors gratefully acknowledge the support by the European Commission under the 'EUIndia Cross Cultural Innovation Network' project.

\section{References}

Brislin, R. and Yoshida, T. (1994), An Introduction to Intercultural Communication Training, Thousand Oaks, CA: Sage.

Conner, D. W. (1990), 'Distance teaching across cultures: implications for instructional design', in Proceedings of the 6th Annual Conference on Distance Teaching and Learning, Madison, WI: University of Wisconsin.

Dron, J., Mitchell, R., Siviter, P. and Boyne, C. (2000), 'Website evaluation system: collaborative discovering what makes a website good', in Proceedings of WebNet 2000, October 2000, AACE.

Dron, J., Mitchell, R., Siviter, P. and Boyne, C. (1999), 'CoFIND - an experiment in ndimensional collaborative filtering', in Proceedings of WebNet 99, Hawaii, October 1999, AACE.

EU-India prototype Website (2000), http://learntech.bton.ac.ukleuindial, URL last accessed 24 December 2001.

Jadhav, L. (1999), South Asian Immigrants - Living in Two Cultures (ERIC Document Reproduction Service no. ED431337).

Moore, M. and Kearsley, G. (1996), Distance Education: A Systems View, Belmont, CA: Wadsworth.

Murphy, K. L. (1991), 'Sociocultural context of Turkish distance learning', in Proceedings of the Seventh Annual Conference on Distance Teaching and Learning, Madison, WI: University of Wisconsin. 